http://jmscr.igmpublication.org/home/ ISSN (e)-2347-176x ISSN (p) 2455-0450 crossref DOI: https://dx.doi.org/10.18535/jmscr/v7i10.23

\author{
Journal Of Medical Science And Clinical Research

\title{
Bronchial Neuroendocrine Tumour in Young Female: A Case Report
}

\author{
Authors \\ Dr Vaishali Walke ${ }^{1}$, Dr Amrapali Gaikwad ${ }^{2}$, Dr Suyog Ratnaparkhi ${ }^{3 *}$, \\ Dr Balwant Kowe \\ ${ }^{1}$ Associate Professor, ${ }^{2}$ Assistant Professor, ${ }^{3}$ Resident Pathology, ${ }^{4}$ Professor and Head \\ Department of Pathology, Indira Gandhi Govt Medical College, Nagpur-440018, Maharashtra, India \\ *Corresponding Author \\ Dr Suyog Ratnaparkhi
}

Plot no. 82, New Indira Colony, Bhagwan Nagar, Near Sainath School, Nagpur- 440027, Maharashtra, India

\begin{abstract}
The bronchopulmonary carcinoids are slow growing neuroendocrine tumours arising from enterochromaffin (EC) cells of bronchial epithelium comprising of $1 \%$ to $2 \%$ of all lung tumours. Central bronchial carcinoids are more common than the peripheral type. They are characterized by strikingly heterogeneous pathological features and clinical behavior. Surgical resection is the preferred treatment for localized carcinoid. Trans bronchoscopic resection may be an alternative for entirely endobronchial lesions. We report a case of 27 year old female presented with chest pain. High Resolution Computed Tomography (HRCT) reveals mass lesion of infective aetiology. Contrast Enhanced Computed Tomography (CECT) showed a well defined neoplastic growth occluding the right main bronchus. The diagnosis of Neuroendocrine Tumour / Carcinoid offered on endoscopic biopsy was confirmed on immunohistochemistry.

Keywords: Bronchial carcinoid, atypical carcinoid, neuroendocrine tumors.
\end{abstract}

\section{Introduction}

Carcinoid tumors are neuroendocrine tumors derived from Enterochromaffin or Kulchitsky cells, which are widely distributed in the body. ${ }^{1}$ They are found usually in small intestine (26\%), respiratory system $(25 \%)$ and appendix $(19 \%){ }^{2}$ Bronchial carcinoid tumours termed (incorrectly) as bronchial adenomas in the past are uncommon pulmonary neoplasms. ${ }^{3}$ They make up 1-2\% of all lung tumors ${ }^{4}$ and often arise in persons who are younger than the one for lung cancers. The male to female ratio is $1: 1$. These are characterized by positive reaction to silver stains and to markers of neuroendocrine tissue, including neuron specific enolase (NSE), synaptophysin and chromogranin. ${ }^{5}$ Early diagnosis is extremely important as patients may benefit from surgical resection and the prognosis is very good.

\section{Case Report}

A, 27 year female presented with breathlessness, cough with expectoration and right sided chest pain since 1 month. His chest X-ray showed a homogenous opacity in upper, middle and lower zone of right lung. Bronchoscopy revealed a polypoidal mass completely obstructing the right 
main bronchus. The High-resolution computed tomography (HRCT) thorax was suggestive of infective etiology with mucus plug in right main bronchus. Contrast enhanced computed tomography (CECT) thorax however revealed mass lesion at right main bronchus suggestive of primary neoplastic etiology. We received bronchoscopic biopsy as a single tissue piece of size $0.8 \times 0.5 \times 0.1 \mathrm{~cm}$, whitish and soft. Histopathology revealed tumor cells arranged in small nests and sheets separated by thin fibrocollagenous tissue. (Figure.1) Individual tumor cells were monotonous, small, round to oval with central round monomorphic nuclei with fine dense chromatin, inconspicuous nucleoli and moderate amount of pale, eosinophilic cytoplasm. Occasional binucleation was also seen. (Figure. 2) Mitotic count was very low $(<2 / 10 \mathrm{hpf})$. There was neither an evidence of anaplasia nor necrosis. Based on morphology the diagnosis of Neuroendocrine Tumour Grade-I - Carcinoid was offered. The panel of IHC markers applied, showed strong cytoplasmic positivity for synaptophysin (Figure 3) and chromogranin A (Figure 4). The tumor cells also expressed cytokeratin and Ki67 index was low (Figure 5), while staining for TTF was negative. The final diagnosis of typical carcinoid (Neuroendocrine tumor Grade-I) was therefore established.

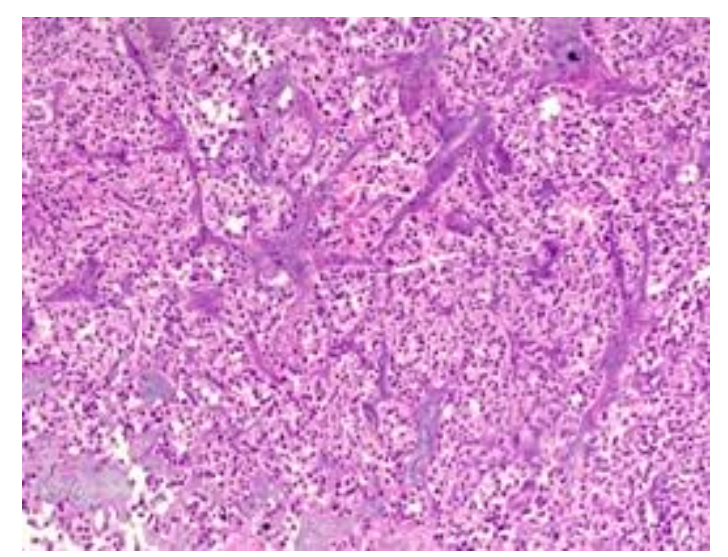

Figure 1: Tumor cells displaying organoid pattern. (HE stain, 10X)

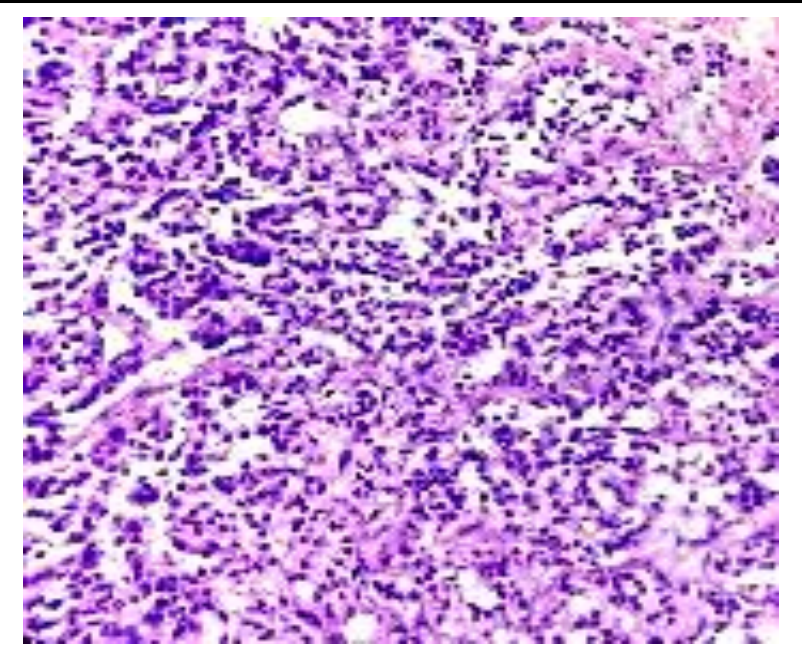

Figure 2: Individual cells: monotonous, round with moderate pale eosinophilic cytoplasm, central round monomorphic nuclei, fine chromatin. (HE stain, 40X)

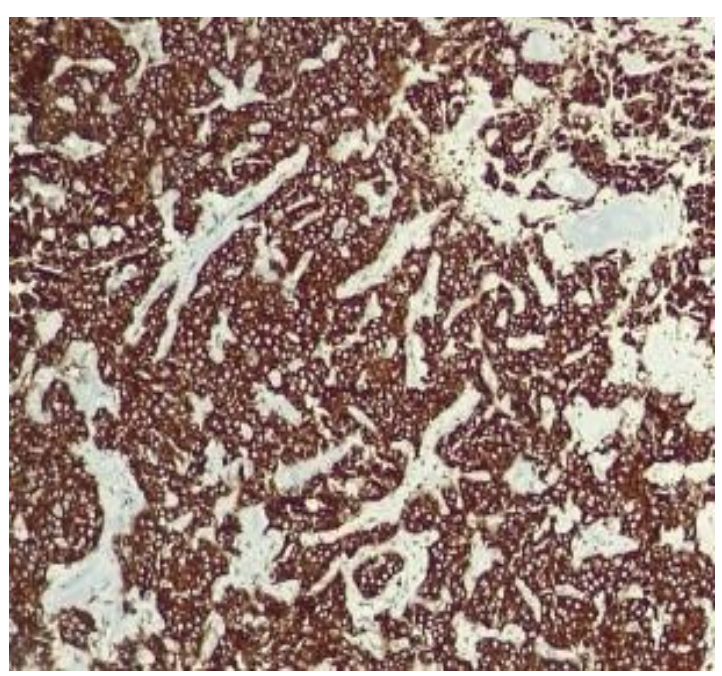

Figure 3: Strong, cytoplasmic positivity in neoplastic cells. (IHC: Synaptophysin, 10X)

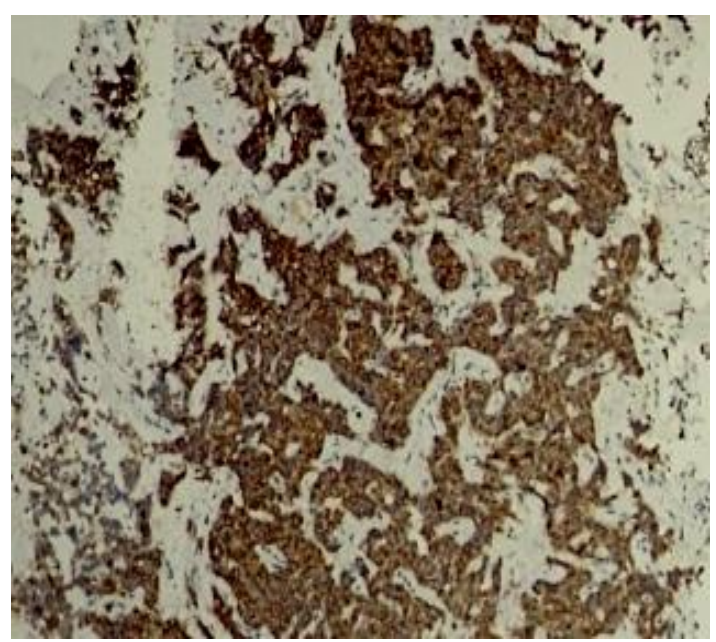

Figure 4: Strong cytoplasmic positivity in neoplastic cells. (IHC: Chromogranin A, 10X) 


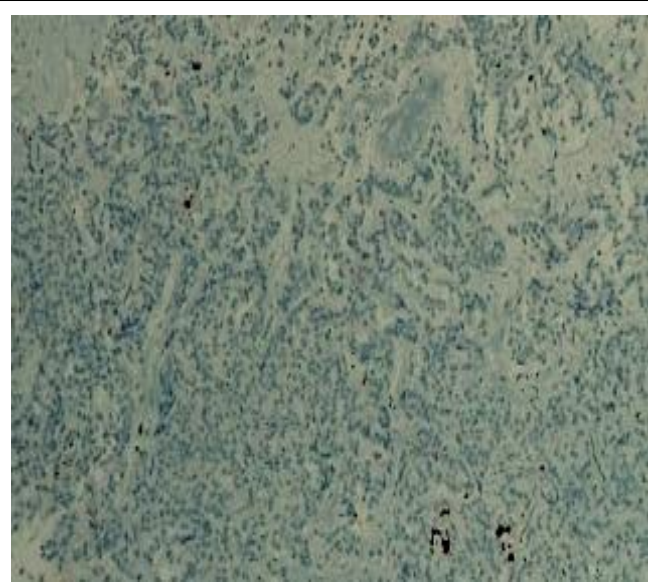

Figure 5: Focal and weak, nuclear staining in tumour cells (Low Index). (IHC: Ki67, 10X)

\section{Discussion}

Bronchial carcinoids are rare tumors representing $0.5-2.5 \%$ of all pulmonary neoplasms. ${ }^{6}$ According to current classification, carcinoids are considered as neuroendocrine tumors (NET) having three grades based on the histologic features and biologic behavior. NET-Grade I is considered as typical carcinoid, Grade II NET as atypical carcinoid and Grade III NET is called small cell carcinoma/large cell carcinoma. ${ }^{7}$ Typical carcinoids occur in both sexes with equal frequency and the age at onset ranges from childhood to 9th decade. A diagnosis of bronchial carcinoids is difficult in young patients as these patients present with non specific symptoms. The symptoms depend on the location of tumor; central or peripheral. Central tumors are mostly located in right lung and cause hemoptysis or recurrent bronchial obstruction as noted in our case. Endocrine manifestations occur in $1-7 \%$ of cases including carcinoid syndrome which occurs in $86 \%$ of patients with liver metastases. ${ }^{8}$ The Chest X-ray may not detect the central lesion due to its smaller size as observed in our case. Contrast-enhanced computerized tomography (CECT) remains a highly sensitive modality of investigation that can easily pick up a small, rounded endobronchial nodule which appears intensely enhancing on contrast. On histology, NET grade I tumors exhibits an organoid pattern and the cells show salt and pepper type of nuclear chromatin. According to recent WHO classification, atypical carcinoid (NET II) differs from typical carcinoid by presence of punctuate coagulative necrosis and or mitotic figure ranging from 2 - 10/10 high-power fields. In both typical and atypical carcinoid, the stroma is vascular. All neuroendocrine tumors irrespective of grade show positively for chromogranin, synaptophysin. It's KI 67 index which helps to differentiate these from one another. In the present case, even on small endoscopic biopsy specimen, we could observe organoid pattern and characteristic nuclear chromatin at places and rare mitosis. The tumor cells expressed both synaptophysin and chromogranin with low Ki 67 index which together helped to arrive at a definitive diagnosis. The closest differential diagnosis includes atypical carcinoid and a wide variety of non neuroendocrine tumors like sclerosing hemangioma, paraganglioma, glomus tumor and adenocarcinoma which may resemble carcinoid. In these situations, morphology in the form of organoid pattern, nuclear chromatin along with expression of neuroendocrine markers can contribute to establish the correct diagnosis.

Biological behavior and prognosis for bronchial carcinoid is excellent as they rarely metastasize to regional lymph nodes, in contrast atypical carcinoids have higher likelihood of distant metastases. Surgical resection is the treatment of choice for typical carcinoid with good long term survival. ${ }^{9,10}$

\section{Conclusion}

In the absence of specific symptoms, bronchial carcinoid poses diagnostic difficulty. In young patient with chest symptoms, endobronchial tumors should be considered in the differential diagnosis apart from more common infective etiologies like pulmonary tuberculosis. A high index of clinical suspicion along with radiological investigations and histopathology is extremely important to establish early and correct diagnosis.

No conflict of interest \& financial support: nil 


\section{References}

1. Zuetenhorst J M, Taal B G. Metastatic carcinoid tumors: A clinical review. Oncologist 2005; 10:123-31.

2. Emeryk J, Czekajska-Chehab E, Korobowicz E, Korbel M, WegrzynSzkutnik I, Milanowski J. Bronchial carcinoid in a 39-year-old man treated for bronchial asthma: A case report. Cases J 2008;2:7414.

3. Davila D G, Dunn W, Tazelaar H D, Pairolero P C. Bronchial carcinoid tumors. Mayo Clin Proc 1993;68:795-803.

4. Hage R, de la Rivière $A B$, Seldenrijk CA, van den Bosch JM. Update inpulmonary carcinoid tumors: A review article. Ann Surg Oncol 2003;10:697-704.

5. Kulke MH, Mayer RJ. Carcinoid tumor. N Engl J Med 1999;340:858-68.

6. Bhatia K, Ellis S. Unusual lung tumors. An illustrated review of CT features suggestive of this diagnosis. Cancer Imaging. 2006;6:72-82.

7. Sadana M J, Mones JM, Garcia-Molinear M, Frable W J. Localized disordersof the bronchi \& lung. In: Silverberg SG, editor. Principles \& Practice of Surgical Pathology \& Cytopathology. 3rd ed. New York, NY: Churchill Livingstone Inc.; 1997. p. 1189-297.

8. Bora MK, Vithiavathi S. Primary bronchial carcinoid: a rare differential diagnosis of pulmonary Koch in young adult patient. Lung India: Official Organ of Indian Chest Society. 2012;29(1):5962.

9. Brambilla E, Lantuejoul S. Neuroendocrine neoplasms. In: Zander D $\mathrm{S}$, Farver C F, editors. Pulmonary Pathology. 1st ed. Philadelphia, PA: Churchill Livingstone, Elsevier; 2008. p. 563-77.
10. Kulkarni G, Gawande S, Chaudhari D, Bhoyar A. Bronchial carcinoid: case report and review of literature. MVP Journal of Medical Sciences. 2016;3(1):71-78. 